\title{
Stoffwechseluntersuchungen bei Mikroorganismen mit Hilfe radioaktiver Isotope IV
}

\author{
Umwandlung von Guanin in Adenin durch Lactobacillus leichmannii 313, \\ untersucht mit Guanin- $\left(8-{ }^{14} \mathrm{C}\right)$ und Adenin- $\left({ }^{14} \mathrm{C}\right)$
}

\author{
Von Friedrich Weygand, Adolf Wacker und Hanswerner Dellweg \\ Aus dem Chemischen Institut der Universität Heidelberg \\ (Z. Naturforschg. 7 b, 156-161 [1952]; eingegangen am 8. Januar 1952)
}

\begin{abstract}
Durch Verwendung von Guanin-(8-14C) konnte bewiesen werden, daß L. leichmannii 313 Guanin in Adenin verwandelt. Umgekehrt wird Adenin-( $\left.{ }^{14} \mathrm{C}\right)$ nicht in Guanin übergeführt. Ferner wurde die Wirkung von nichtradioaktivem Adenin, Xanthin und Hypoxanthin auf die Umwandlungsrate von Guanin in Adenin untersucht.
\end{abstract}

$\mathrm{D}$ urch Anwendung der Hemmstoffanalyse (inhibition analysis) kamen wir zu der Auffassung, daß Lactobacillus leichmannii 313 Guanin in Adenin verwandelt, nicht dagegen Adenin in Guanin ${ }^{1}$. Um dies definitiv zu beweisen, führten wir Versuche mit Guanin- $\left(8-{ }^{14} \mathrm{C}\right)$ und Adenin- $\left({ }^{14} \mathrm{C}\right)$ durch. Eine verbesserte Synthese für Guanin- $\left(8-{ }^{14} \mathrm{C}\right)$ ist von uns angegeben worden ${ }^{2}$. Als radioaktives Adenin kam dasjenige zur Verwendung, das die Bakterien aus Guanin gebildet hatten. Es war durch Papierchromatographie aus dem Bakterienhydrolysat abgetrennt worden und stellt mit großer Wahrscheinlichkeit Adenin- $\left(8-{ }^{14} \mathrm{C}\right)$ dar.

\section{Methodik}

\section{A. Züchtung der Bakterien}

S t a m m: Lactobacillus leichmannii 313. Züchtung, Vorzüchtung, Sterilisation, Impfmenge, Bebrütung und Messung des Bakterienwachstums sind in einer früheren Arbeit angegeben worden ${ }^{1}$. Dort ist auch das verwendete Nährmedium beschrieben. Die Züchtung wurde in 100ccm-Erlenmeyerkolben mit $\mathrm{K}$ a p s e $\mathrm{n}$ b e r g-Kappen durchgeführt. Jede Versuchsreihe wurde doppelt oder 3-fach angesetzt. Als Pyrimidinquelle enthielt das Nährmedium bei jedem Versuch $10 \gamma / \mathrm{ccm}$ Uracil. Die Mengen an Purinen sowie an Wuchs- und Hemmstoffen sind in den Tabellen aufgeführt.

Die Bakterien wurden bebrütet, bis maximales Wachstum erreicht war, wozu 48-72 Stdn. erforderlich waren. Nach Aufschütteln der gewachsenen Bakterien wurde jeweils ein Teil der Kultur in ein Reagensglas für mikrobiologische Zwecke umgefüllt und der Trübungswert im

1 F. W e y g a nd, A. W a c k e r u. F. Wirth, Z. Naturforschg. 6 b, 25 [1951].

2 F. Weygand u. O. A. Großkinsky, Chem. Ber. 84, 839 [1951]. lichtelektrischen Kolorimeter nach Dr. B. L a nge bestimmt. Zur Kontrolle des Wuchsstoffbedürfnisses von L. leichmannii 313 wurden bei allen Ansätzen Hemmund Enthemmungsversuche mit 5-Methyl-4-amino-2-thiouracil (Mathu), Vitamin $\mathrm{B}_{12}$ und Desoxyribosiden mitgeführt ${ }^{1}$.

\section{B. Aufarbeitung der Bakterien}

Zur Isolierung der Bakterien wurde die Kulturlösung zentrifugiert. Die mit Wasser gewaschenen Bakterien wurden von säurelöslichen, niedrigmolekularen Nucleotiden mit eiskalter wäßriger Trichloressigsäure befreit und anschließend mit einer heißen Alkohol-Äther-Mischung entfettet ${ }^{3,9}$ (vgl. B ${ }_{1}$ ). In Anbetracht der zur Verfügung stehenden geringen Bakterienmenge verzichteten wir auf eine Isolierung der Nucleinsäuren und behandelten die Bakterien zur Freilegung der Purine und Pyridine direkt mit Perchlorsäure nach den Angaben von $\mathrm{M}$ arskak und V o g e ${ }^{4}\left(\right.$ vgl. $\left.B_{2}\right)$. Die so erhaltene Lösung wurde an Papier chromatographiert, wobei es sich als zweckmäßig erwiesen hat, vor dem Aufsetzen auf das Chromatogramm die Perchlorsäure mit Kalilauge zu neutralisieren. Nach dem Entwickeln wurden die Purine und Pyrimidine auf dem Papier nach der Methode von $\mathrm{M}$ a r kham und $\mathrm{S} \mathrm{mith}{ }^{5}$ nachgewiesen (vgl. $\left.\mathrm{B}_{3}\right)$. Danach eluierten wir die Purine und bestimmten die Konzentration von Guanin und Adenin durch Kolorimetrie im Ultravioletten (vgl. $\mathrm{B}_{5}$ ).

3 S. E. K err, K. S e raid a rian u. G.B.Brown, J. biol. Chemistry 188, 207 [1951].

4 A. M a r s a k u. H. J. V o g e l, J. biol. Chemistry 189, 597 [1951].

5 R. M a r kham u. J. D. S m ith, Biochem. I. 45. 294 [1949]. 
B. $_{1}$ Gewinnung der B a k terien: Der Inhalt von 2 bzw. 3 Kolben (je $60 \mathrm{ccm}$ Kulturflüssigkeit) wurde vereinigt, worauf die Bakterien abzentrifugiert wurden (15000 Umdr./min). Dann wurden sie in den nachfolgenden Reagenzien aufgeschlämmt und jeweils wieder zentrifugiert:

1. Wasser . . . . . . . . . $10 \mathrm{ccm}$ bei $20^{\circ}$

2. 95-proz. Äthanol . . . . . . . . $10 \mathrm{ccm}$ bei $20^{\circ}$

3. 4. 5. 5-proz.wässrige Trichloressigsäure $5 \mathrm{ccm}$ bei $2^{\circ}$

6. 95-proz. Äthanol . . . . . . . . $10 \mathrm{ccm}$ bei $20^{\circ}$

7. 8. 9. Äthanol-Äther $(3: 1)$. . . . . $10 \mathrm{ccm}$ siedend

10. Äther . . . . . . . . . $10 \mathrm{ccm}$ bei $20^{\circ}$

Schließlich wurden die Bakterien im Vakuumexsikkator 24 Stdn. getrocknet. Ausb.: 55-75 mg getrocknete und entfettete Bakterien aus $120 \mathrm{ccm}$ Kulturflüssigkeit.

B $_{2}$. H y droly s e : Etwa $40-45 \mathrm{mg}$ getrocknete Bakterien wurden in ein graduiertes Zentrifugenglas eingewogen, mit $0,16 \mathrm{ccm} 70$-proz. $\mathrm{HClO}_{4}$ versetzt und 1 Stde. im offenen Röhrchen unter gelegentlichem Umrühren auf dem siedenden Wasserbad erhitzt. Nach dem Abkühlen verdünnten wir die dunkelbraune Flüssigkeit auf $0,5 \mathrm{ccm}$, neutralisierten mit $\mathrm{KOH}$ und füllten auf $1 \mathrm{ccm}$ auf. Die überstehenden gelbe bis dunkelbraune Lösung konnte nach dem Abzentrifugieren von dem aus $\mathrm{KClO}_{4}$ und Verkohlungsprodukten bestehenden Rückstand dekantiert werden.

B $_{3}$. Chromatographische Trennung der Spaltprodukte: Die in dieser Lösung enthaltenen Purine und Pyrimidine wurden papierchromatographisch getrennt. Papier: Whatmann Nr. 1, $35 \times 60 \mathrm{~cm}$. Lösungsmittel: $\boldsymbol{n}$-Butanol, mit Wasser gesättigt. Es wurde absteigend bei $20^{\circ}$ chromatographiert. Laufzeit etwa $20 \mathrm{Stdn}$., Entfernung Startlinie-Lösungsmittelfront $40-50 \mathrm{~cm}$. Von der Hydrolysenlösung setzten wir je 0,04, 0,06, 0,08 und $0,10 \mathrm{ccm}$ mit einem Zwischenraum von $3 \mathrm{~cm}$ auf die Startlinie des Chromatogramms. Die Länge der Flecken betrug in jedem Fall $2 \mathrm{~cm}$. Um z. B. $0,08 \mathrm{ccm}$ auf eine Länge von $2 \mathrm{~cm}$ aufzuteilen, mußten 4 -mal je $0,02 \mathrm{ccm}$ aufgesetzt werden, wobei zwischendurch mit warmer Luft angetrocknet wurde. Zur Bestimmung der Radioaktivität der den Bakterien angebotenen markierten Purine wurden bei jedem Chromatogramm $20 \gamma$ in der gleichen Weise auf einen Fleck aufgesetzt.

Anf den entwickelten und getrockneten Chromatogrammen wurden die Stellen, an denen sich die Purine und Pyrimidine befanden, durch Photographie im UVLicht $(\sim 260 \mathrm{~m} \mu)$ ermittelt 5 .

$\mathbf{B}_{4}$. Radioaktive M e s s ungen: Nach der Übertragung der photographischen Aufnahme auf das Papier wurden die einzelnen Flecken in Rechtecken zu je $5 \times 2$ bzw. $5 \times 4 \mathrm{~cm}$ ausgeschnitten. Die Aktivität der einzelnen Streifen bestimmten wir, indem wir diese ganz oder in zwei Hälften unter eine rechteckige Metallblende $(20 \times 38 \mathrm{~mm})$ schoben und die ausgesandte Strahlung mit einem $\beta$-Zählrohr $\left(7 \mathrm{mg} / \mathrm{cm}^{2}, 16 \times 31 \mathrm{~mm}\right)$ und dem Strahlungsmeßgerät FH 41 der Fa. Fri es e c k e \& H o e p fner, Erlangen-Bruck, maßen. In fast allen Fällen fanden wir eine mehr oder weniger große Aktivität auf der Startlinie, die dem nicht vollständig abgewanderten Guanin zugeschrieben werden muß. Auf die Ermittlung der molaren Aktivität hat diese Erscheinung keinen Einfluß. Sie muß lediglich bei der Bestimmung der pro mg Bakterien enthaltenen Menge Guanin berücksichtigt werden. Die Pyrimidine waren in keinem. Fall radioaktiv.

$B_{5}$. Ermittlung der Purinmengen durch UV-S pektrophot ometrie. Nach der Radioaktivitätsbestimmung wurden die Papierstreifen zerschnitten und in Reagensgläsern mit je $5 \mathrm{ccm} 0,1-n . \mathrm{HCl}(1-n . \mathrm{HCl}$, M erck, verdünnt mit doppelt dest. Wasser) übergossen. In der gleichen Weise wurden auch entsprechende Papierstreifen behandelt, auf denen keine Substanz gewandert war; die so erhaltene Lösung diente als Füllung für die Vergleichsküvette. Die Röhrchen ließen wir unter gelegentlichem Umschütteln $20 \mathrm{Stdn}$. bei $37^{\circ}$ stehen und füllten die Eluate durch vorsichtiges Dekantieren in die Meßküvetten. Das UV-Spektrum bzw. ein Teil desselben, wurde im B e c k m a n - Spektrophotometer, Modell DU, aufgenommen. Aus der Höhe des Absorptionsmaximums wurde durch Vergleich mit chromatographierten Lösungen bekannten Gehaltes die Konzentration des betreffenden Purins ermittelt. Aus der Konzentration und der Aktivität des Papierstreifens läßt sich so die Aktivität in Impulsen $/ \mu \mathrm{Mol} / \mathrm{min}$ errechnen.

$\mathbf{B}_{6}$. Fehlerdiskussion. Bei den aufgeführten Arbeitsweisen sind folgende Fehlerquellen zu berücksichtigen:

1. Einwaagefehler und Ungenauigkeit beim Auffüllen auf $1 \mathrm{ccm}$.

2. Fehlerhaftes Aufsetzen der Lösungen auf das Papierchromatogramm mit der Mikropipette.

3. Unvollständige Trennung der Substanzen auf dem Chromatogramm.

4. Fehler bei der Messung der Radioaktivität. Je nach der Aktivität der Papierstreifen wählten wir die Meßzeit so groß, daß mindestens 1000 Teilchen gezählt wurden; die Meßzeit betrug mindestens $10 \mathrm{Mi}-$ nuten.

5. Fehler bei der Elution der Papierstreifen.

6. Fehler bei der UV-Spektrometrie. Der Gerätefehler beträgt $0,5-1 \%$.

Unter der Annahme der ungünstigsten Verhältnisse, d. h. wenn alle Fehler das Endergebnis im gleichen Sinne beeinflussen, ergibt sich der größtmögliche Gesamtfehler des Endergebnisses zu $\pm 10-12 \%$. Da wir jeweils vier Parallelbestimmungen mit verschiedenen Konzentrationen ausführten, sind in den Abweichungen dieser 4 Ergebnisse vom Mittelwert sämtliche unter 2-6 aufgeführten Fehler enthalten. Der Errechnung des wahrscheinlichen Fehlers der Messungen legten wir die Formel

$$
N=N_{m} \pm \sqrt{\frac{\sum_{i}\left(N_{m}-N_{i}\right)^{2}}{n-1}}
$$

zugrunde, in der

$N_{i}$ das Resultat einer Messung,

$N_{m}$ das arithmetische Mittel aller Messungen und

$n$ die Anzahl der Messungen bedeuten. 


\begin{tabular}{|c|c|c|c|c|c|c|c|c|c|c|c|c|c|c|c|c|c|}
\hline \multirow[b]{3}{*}{$\mathrm{Nr}$} & \multicolumn{9}{|c|}{$\gamma / \mathrm{cm}^{3}$ Nährmedium } & \multicolumn{8}{|c|}{ Isolierte Purine } \\
\hline & \multirow[b]{2}{*}{ 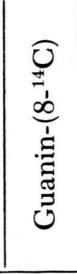 } & \multirow[b]{2}{*}{ 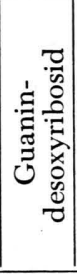 } & \multirow[b]{2}{*}{ } & \multirow[b]{2}{*}{ 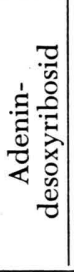 } & \multirow[b]{2}{*}{ 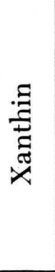 } & \multirow[b]{2}{*}{ 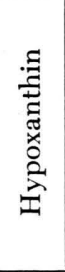 } & \multirow[b]{2}{*}{ 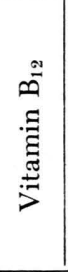 } & \multirow[b]{2}{*}{ 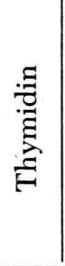 } & \multirow[b]{2}{*}{ 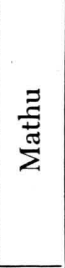 } & \multicolumn{4}{|c|}{ Guanin } & \multicolumn{4}{|c|}{ Adenin } \\
\hline & & & & & & & & & & 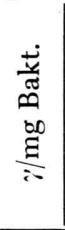 & 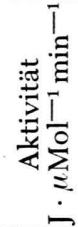 & $\div$ & $\frac{\frac{d}{0}}{\frac{0}{0}}$ & 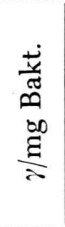 & 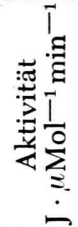 & $\stackrel{2}{a}$ & 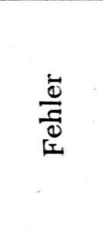 \\
\hline 1 & 2 & 3 & 4 & 5 & 6 & 7 & 8 & 9 & 10 & 11 & 12 & 13 & 14 & 15 & 16 & 17 & 18 \\
\hline I a & 13,3 & & & & & & 0,33 & & & & 3920 & 82,0 & $\pm 6,14$ & 4,2 & 3810 & 81,0 & $\pm 3,46$ \\
\hline I b & $\begin{array}{l}13,3 \\
13,3 \\
13,3\end{array}$ & & $\begin{array}{l}1,6 \\
3,3 \\
6,6\end{array}$ & & & & $\begin{array}{l}0,33 \\
0,33 \\
0,33\end{array}$ & & & $\begin{array}{l}5,0 \\
5,4 \\
4,7\end{array}$ & $\begin{array}{l}4230 \\
4295 \\
3790\end{array}$ & $\begin{array}{l}78,5 \\
79,5 \\
80,5\end{array}$ & $\begin{array}{r} \pm 3,58 \\
\pm 3,46 \\
\pm 2,06\end{array}$ & $\begin{array}{l}4,1 \\
4,4 \\
4,6\end{array}$ & $\begin{array}{r}2640 \\
1772 \\
725\end{array}$ & $\begin{array}{l}49,0 \\
32,8 \\
15,4\end{array}$ & $\begin{array}{l} \pm 1,36 \\
\pm 0,92 \\
\pm 1,1\end{array}$ \\
\hline I c & $\begin{array}{l}13,3 \\
13,3\end{array}$ & 6,6 & & 6,6 & & & $\begin{array}{l}0,33 \\
0,33\end{array}$ & & & $\begin{array}{l}6,6 \\
7,4\end{array}$ & $\begin{array}{l}3590 \\
4100\end{array}$ & $\begin{array}{l}66,5 \\
76,0\end{array}$ & $\begin{array}{r} \pm 2,99 \\
\pm 2,93\end{array}$ & $\begin{array}{l}4,6 \\
5,5\end{array}$ & $\begin{array}{l}3480 \\
1607\end{array}$ & $\begin{array}{l}64,5 \\
29,8\end{array}$ & $\begin{array}{l} \pm 3,26 \\
\pm 1,09\end{array}$ \\
\hline II & \begin{tabular}{|l|}
13,3 \\
13,3 \\
13,3 \\
13,3
\end{tabular} & & $\begin{array}{l}1,6 \\
3,3 \\
6,6\end{array}$ & & & & $\begin{array}{l}0,33 \\
0,33 \\
0,33 \\
0,33\end{array}$ & & $\begin{array}{r}66 \\
266 \\
266 \\
66 \\
\end{array}$ & $\begin{array}{l}4,9 \\
6,6 \\
8,6 \\
9,2 \\
\end{array}$ & $\begin{array}{l}4445 \\
4450 \\
4745 \\
4080\end{array}$ & $\begin{array}{l}94,5 \\
82,4 \\
87,9 \\
86,7 \\
\end{array}$ & $\begin{array}{l} \pm 4,45 \\
\pm 3,61 \\
\pm 3,05 \\
\pm 1,98\end{array}$ & $\begin{array}{l}3,8 \\
4,2 \\
5,5 \\
5,7 \\
\end{array}$ & $\begin{array}{r}4717 \\
3498 \\
2170 \\
734\end{array}$ & $\begin{array}{l}100 \\
64,7 \\
40,1 \\
15,6 \\
\end{array}$ & $\begin{array}{l} \pm 2,45 \\
\pm 1,79 \\
\pm 2,09 \\
\pm 0,7\end{array}$ \\
\hline III & $\begin{array}{l}13,3 \\
13,3 \\
\end{array}$ & & & & 6,6 & 6,6 & $\begin{array}{l}0,33 \\
0,33\end{array}$ & & & $\begin{array}{l}7,4 \\
7,0\end{array}$ & $\begin{array}{l}3645 \\
3170\end{array}$ & $\begin{array}{l}67,5 \\
58,0\end{array}$ & $\begin{array}{l} \pm 3,8 \\
\pm 1,83\end{array}$ & $\begin{array}{l}5,0 \\
4,7\end{array}$ & $\begin{array}{l}1890 \\
3.805\end{array}$ & $\begin{array}{l}35,0 \\
70,5\end{array}$ & $\begin{array}{l} \pm 2,1 \\
\pm 1,90\end{array}$ \\
\hline IV & $\begin{array}{l}13,3 \\
13,3 \\
13,3\end{array}$ & & $\begin{array}{l}6,6 \\
6,6\end{array}$ & & & & & $\begin{array}{l}6,6 \\
6,6 \\
6,6\end{array}$ & 66 & $\begin{array}{l}7,0 \\
9,3 \\
7,2\end{array}$ & $\begin{array}{l}3890 \\
4070 \\
4000\end{array}$ & $\begin{array}{l}82,6 \\
86,5 \\
85,0\end{array}$ & $\begin{array}{l} \pm 2,53 \\
\pm 1,43 \\
\pm 1,9\end{array}$ & $\begin{array}{l}4,7 \\
6,7 \\
4,0\end{array}$ & $\begin{array}{r}4230 \\
1011 \\
758\end{array}$ & $\begin{array}{l}90,9 \\
21,5 \\
16,1\end{array}$ & $\begin{array}{l} \pm 3,03 \\
\pm 1,17 \\
\pm 0,72\end{array}$ \\
\hline
\end{tabular}

Tab. 1. Zusammenstellung der Ergebnisse.

Spalte 2-10: Die dem Nährmedium zugesetzten Verbindungen in $\gamma / \mathrm{cm}^{3}$; Spalte 8 in $\mathrm{m} \gamma / \mathrm{cm}^{3}$. Spalte 11 und 15 : Menge der pro mg getrockneter und entfetteter Bakterien enthaltenen Purine in $\gamma$. Spalte 12 und 16: Aktivität der isolierten Purine in Impulsen $\cdot \mu \mathrm{Mol}-1 \cdot \min ^{-1}$. Spalte 13 und 17: Aktivität der isolierten Purine in \% der Aktivität des eingesetzten Purins-14C. Spalte 14 und 18: Wahrscheinlicher Fehler der Werte in Spalte 13 und 17.

\begin{tabular}{|c|c|c|c|c|c|c|c|c|c|c|c|}
\hline & Adenin- $\left({ }^{14} \mathrm{C}\right)$ & Guanin & $\begin{array}{l}\text { Vit. } \\
\mathbf{B}_{12}\end{array}$ & \multicolumn{8}{|c|}{ Siehe oben } \\
\hline 1 & 2 & 4 & 8 & 11 & 12 & 13 & 14 & 15 & 16 & 17 & 18 \\
\hline & 1,25 & 0,83 & 0,33 & 4,8 & 0 & 0 & - & 3,6 & 1609 & 46,0 & $\pm 1,88$ \\
\hline
\end{tabular}

T'ab. 2. Erklärung der einzelnen Spalten s. Tab. 1.

Für die Angabe Impulse $/ \mu \mathrm{Mol} / \mathrm{min}$ fanden wir auf diese Weise einen Fehler, welcher sich zwischen \pm 2 und $9 \%$ bewegt.

Auf die Bestimmung der molaren Aktivität durch direktes Auszählen der Papierstreifen sei noch kurz eingegangen. ${ }^{14} \mathrm{C}$. ist ein weicher $\beta$-Strahler $\left(E_{\max }=0,154 \mathrm{MeV}\right)$. Die Selbstabsorption im Papier ist bei unserer Art der Messung sehr groß (schätzungsweise 90\%). Da nur das Verhältnis der Aktivitäten der gefundenen zu der der eingesetzten Substanzen von Interesse ist, ist diese Arbeitsweise dann berechtigt, wenn man auch die Aktivität der dem Nährmedium zugesetzten Substanzen auf dem gleichen Wege bestimmt. Dabei setzten wir allerdings gleiche Beschaffenheit und Dicke des zur Chromatographie verwendeten Papiers in einem Bogen voraus.

\section{Ergeb nis se (vgl. Tab. 1).}

Bei Züchtung von L. leichmannii 313 mit Guanin${ }^{8-14} \mathrm{C}$ als einziger Purinquelle ist das von den Bakterien gebildete Adenin radioaktiv, und zwar zeigen das aus den Bakterien isolierte Guanin und Adenin nahezu die gleiche Aktivität von $81-82 \%$, bezogen auf die des eingesetzten Guanins gleich 100\%. Bei Zusatz von steigenden Mengen inaktivem Adenin zum Medium verringert sich die Aktivität des isolierten Adenins, ein Zeichen, daß das zugesetzte Adenin in die Nucleinsäure eingebaut wird, während die Aktivität des isolierten Guanins praktisch gleichbleibt. Wird an Stelle von Adenin Adenindesoxy- 
ribosid angeboten, so verringert sich die Aktivität des isolierten Adenins ebenfalls. Wird neben dem radioaktiven Guanin inaktives Guanindesoxyribosid dem Medium zugefügt, so sinkt die Aktivität von Guanin und Adenin in gleichem Maße ab.

Auffallend ist, daß bei Zusatz des Hemmstoffes 5-Methyl-4-amino-2-thio-uracil (Mathu) ${ }^{1}$ die Aktivität des isolierten Guanins und Adenins höher liegt als im Versuch ohne Mathu. Diese Tatsache beobachtet man auch dann, wenn man dem Nährmedium steigende Mengen nichtradioaktiven Adenins zusetzt, wobei die Aktivität des aus den Bakterien isolierten Adenins immer um 20\% höher liegt als bei den Versuchen ohne Mathu. (Die verwendeten Mengen Mathu waren so hoch, daß in Abwesenheit von Vitamin $\mathrm{B}_{12}$ oder Adenin das Bakterium total gehemmt worden wäre.)

Setzt man dem Nährmedium inaktives Hypoxanthin zu, so verringert sich die Aktivität von Adenin stärker als die von Guanin. Inaktives Xanthin bewirkt den umgekehrten Effekt, so daß in diesem Falle das isolierte Adenin eine größere molare Aktivität besitzt als Guanin.

Wird Thymidin als Wuchsstoff verwendet, so ergibt sich etwa dasselbe Bild wie mit Vitamin $\mathrm{B}_{12}$ als Wuchsstoff.

Bei der Prüfung der Frage, ob L. leichmannii 313 Adenin in Guanin verwandeln kann oder nicht, war zunächst folgendes zu bedenken. Da das Bakterium zum maximalen Wachstum Guanin benötigt, und da ferner Adenin in Abwesenheit von Guanin im Nährmedium das Wachstum hemmt ${ }^{1}$, mußten wir bei dem Versuch mit Adenin- $\left({ }^{14} \mathrm{C}\right)$ auch Guanin zusetzen. Wir wählten das Konzentrationsverhältnis so, daß bei einer möglichst großen Adeninkonzentration die Guaninkonzentration gerade eben zur Enthemmung ausreichte. Wie aus Tab. 2 hervorgeht, ist das isolierte Guanin bei Angebot von Adenin- $\left({ }^{14} \mathrm{C}\right)$ nicht radioaktiv. Die Aktivität des isolierten Adenins ist kleiner als die des angebotenen, da Adenin auch aus Guanin entsteht.

\section{Diskussion}

Durch die hier beschriebenen Versuche konnten wir zunächst die Ergebnisse der Hemmstoffanalyse, nämlich die irreversible Umwandlung von Guanin in Adenin, bei L. leichmannii 313 voll bestätigen. Die Tatsache, daß das isolierte Guanin und das Adenin etwa $80 \%$ der Aktivität des eingesetzten Guanins haben, kann folgendermaßen [(a) oder (b)] erklärt werden: (a) Es findet eine gewisse, aber suboptimale Eigensynthese von Guanin durch das Bakterium statt. $\mathrm{Da} \beta$ das Bakterium etwas Guanin selbst synthetisieren kann, ging auch schon daraus hervor, daß bei Abwesenheit von Purinen im Nährmedium nach längerer Bebrütungszeit ein geringes Wachstum stattfindet (l. c. 1, Abb. 1). Diese Eigensynthese würde unter den von uns vorgegebenen Bedingungen etwa 20\% des Bedarfs an Guanin decken. Das so verdünnte Guanin wird z. Tl. in Adenin verwandelt, so daß zwangsläufig das isolierte Adenin die gleiche Aktivität von etwa $80 \%$, bezogen auf das eingesetzte Guanin, hat.

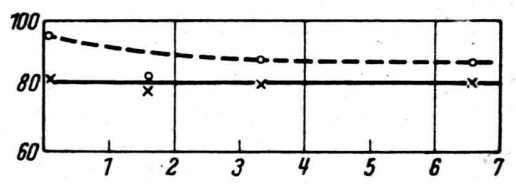

Abb. 1. Aktivität des aus den Bakterien isolierten Guanins in Abhängigkeit von der im Nährmedium befindlichen Konzentration an nichtradioaktivem Adenin. $x-x$ in Abwesenheit von $\mathrm{Mathu}$, $\bigcirc-\circ$ in Anwesenheit von Mathu (Konz. vgl. Tab. 1). Ordinate: Aktivität des isolierten Guanins in \% (eingesetztes Guanin $=100 \%$ ). Abszisse: Konzentration des Adenins im Nährmedium in $\gamma / \mathrm{cm}^{3}$.

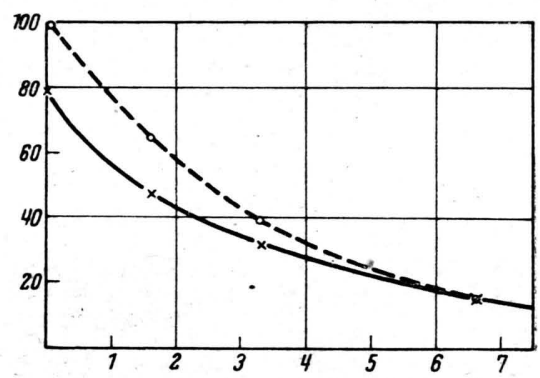

Abb. 2. Aktivität des aus den Bakterien isolierten Adenins in Abhängigkeit von der im Nährmedium befindlichen Konzentration an nichtradioaktivem Adenin. Zeichenerklärung wie in Abb. 1. Ordinate: Aktivität des isolierten Adenins in \% (eingesetztes Guanin = 100\%). Abszisse: s. Abb. 1.

(b) Es könnte auch in Erwägung gezogen werden, was allerdings unwahrscheinlich ist, daß das Bakterium das angebotene Guanin vor seinem Einbau in die Nucleinsäure über eine Zwischenstufe führt, bei der ein Austausch des 8-14C-Atoms gegen inaktiven $\mathrm{C}$ stattfindet.

Entweder muß die Eigensynthese (a) oder der Austausch (b) durch Mathu gehemmt werden, wodurch es zum Ansteigen der molaren Aktivitäten der beiden isolierten Purine kommt (vgl. Tab. 1, Zeile II).

Setzt man dem Medium außer Guanin- $\left(8-{ }^{-14} \mathrm{C}\right)$ auch noch steigende Mengen Adenin zu, so bleibt die Aktivität des isolierten Guanins im wesentlichen gleich (vgl. Abb. 1, untere Kurve), während die des isolierten Adenins kontinuierlich abnimmt (vgl. Abb. 2, un- 
tere Kurve). Demnach wird das von außen zugeführte Adenin vom Bakterium aufgenommen und für den Aufbau der Nucleinsäure verwendet, es. wird aber nur als Adenin eingebaut. Wenn eine Umwandlung von Adenin in Guanin stattfinden würde, so müßte in diesen Fällen die Aktivität des isolierten Guanins ebenfalls absinken.

In Gegenwart von Mathu ist die Aktivität des Guanins nur wenig größer als bei Abwesenheit von Mathu (vgl. Abb. 1, ober Kurve). Die Aktivität des isolierten Adenins liegt in Gegenwart von Mathu um etwa $20 \%$ höher als beim Versuch ohne Mathu; das ist auch dann noch der Fall, wenn im Medium Adenin vorgegeben wird (vgl. Abb. 2, ober Kurve).

Guanindesoxyribosid wird in der gleichen Weise vom Bakterium verwendet wie die freie Base. In Versuch Ic, Tab. 1, enthält das Medium 6,6 $\gamma / \mathrm{ccm}$ Guanindesoxyribosid; das entspricht 3,5 $\gamma$ Guanin. Aus der so entstandenen Verdünnung berechnet sich für das eingesetzte Guanin eine Aktivität von $4280 \mathrm{Imp} \cdot \mu \mathrm{Mol}^{-1} \cdot \mathrm{min}^{-1}$. Nimmt man an, daß wieder $81 \%$ des isolierten Guanins und Adenins aus dem Nährmedium stammen, so müssen diese beiden Verbindungen eine Aktivität von $3470 \mathrm{Imp} \cdot \mu \mathrm{Mol}^{-1}$ $\cdot \min ^{-1}$ besitzen. Diese Zahl deckt sich gut mit den gefundenen von 3590 bzw. 3480 .

Bietet man dem Bakterium Adenindesoxyribosid an, so wird dieses wie Adenin verwertet. Die eingesetzten 6,6 $\gamma / \mathrm{ccm}$ Adenindesoxyribosid (Zeile I c, Tab. 1) enthalten 3,55 $\gamma$ freie Base. Der unteren Kurve der Abb. 2 entnimmt man, daß in diesem Falle das isolierte Adenin $30 \%$ der Aktivität des eingesetzten Guanins besitzen müßte. Gefunden wurden $29,8 \%$.

Es erhebt sich in diesem Zusammenhang die Frage, ob die dem Bakterium angebotenen Desoxyriboside als solche verwertet werden, oder ob sie eine Aufspaltung in die freien Basen und eine DesoxyriboseVerbindung ${ }^{6}$ erleiden. Diese Spaltstücke wären im letzteren Falle für die beobachteten Effekte verantwortlich zu machen, nämlich:

1. Guanindesoxyribosid und Adenindesoxyribosid haben die gleiche molare Wirkung wie die freien Basen bei den oben angeführten Versuchen (Tab. 1, Zeile Ic). Aus diesen Verbindungen würden die

6 Vgl. hierzu die Arbeiten: W. S. M a c N u t t, Nature [London] 166, 444 [1950]; M. F ri ed k i n u. H. M. Ka lck a r, J. biol. Chemistry 184, 437 [1950]; M. Frie d$\mathrm{k}$ i n , ebenda 184, 449 [1950]; E. H of f - J ø r g e n s e n, M. F riedkin u. H. M. K a lck a r, ebenda 184,461 [1950]. freien Purine entstehen, welche die Verdünnung der radioaktiven Purine bewirken.

2. Alle Desoxyriboside, auch Uracildesoxyribosid ${ }^{7}$, das offenbar kein Bestandteil der Desoxyribonucleinsäure ist, sind Wuchsstoffe für L. leichmannii 313 und haben etwa die gleiche molare Aktivität. Daraus kann man schließen, daß bei der Aufspaltung eine Desoxyribose-Verbindung entsteht, die allein die Wuchsstoffaktivität dieser Verbindungen bedingt.

3. In Abwesenheit von Guanin wirkt jedoch Adenindesoxyribosid als Hemmstoff, weil das abgespaltene Adenin unter diesen Bedingungen hemmt ${ }^{1}$.

4. Adenin hebt kompetitiv die Hemmwirkung von Benzimidazolen auf ${ }^{1}$. In gleicher Weise wirkt Adenindesoxyribosid ${ }^{1}$ (wie auch die übrigen natürlich vorkommenden Adeninverbindungen ${ }^{1}$ ), was darauf zurückzuführen ist, daß eine Abspaltung der freien Base erfolgt.

Aus diesen Feststellungen ergibt sich auch eine Erklärung für die früher mitgeteilte Beobachtung 1 , daß verschiedene Benzimidazole, die als solche das Wachstum von L. leichmannii 313 hemmen, dann keine Hemmstoffe mehr sind, wenn sie als $\mathrm{N}$-Glykoside (Glucoside, Xyloside) angeboten werden. Offenbar ist das Fermentsystem, welches die Spaltung der natürlichen Desoxyriboside und Riboside bewirkt, nicht in der Lage, diese unnatürlichen Benzimidazolglykoside zu spalten.

Daß sowohl Hypoxanthin als auch Xanthin als Vorstufe für Guanin und Adenin dienen können, geht aus den Werten des Versuches III, Tab. 1, hervor. Die Umwandlung von Xanthin in Guanin geschieht rein formal über den Austausch einer OH-Gruppe durch $\mathrm{NH}_{2}$, während die Umwandlung in Adenin außerdem noch die Abspaltung der $\mathrm{OH}$-Gruppe in 2-Stellung erfordert. Die Reaktion Xanthin $\rightarrow$ Guanin könnte daher mit größerer Geschwindigkeit verlaufen als die Reaktion Xanthin $\rightarrow$ Adenin, was aber noch nicht die interessante Tatsache erklärt, daß die Aktivität des isolierten Guanins kleiner ist als die des isolierten Adenins. Dies läßt sich vorläufig nur so erklären, daß das aufgenommene Xanthin in gebundener Form in erster Linie in Guanin, in geringerem Maße auch in Adenin verwandelt wird. Das auf diese Weise entstandene Guanin dürfte im Gegensatz zu dem von außen aufgenommenen freien Guanin nicht mehr in Adenin verwandelt werden.

Bei der Umwandlung von Hypoxanthin in Adenin und Guanin liegen die Verhältnisse gerade umgekehrt, d.h. der Austausch der $\mathrm{OH}-\mathrm{Gruppe}$ gegen eine Aminogruppe (Hypoxanthin $\rightarrow$ Adenin) könnte

7 F. Weygand, A. W a cker u. H. Dellweg, Z. Naturforschg. 6 b, 130 [1951]. 
rascher erfolgen als der Einbau einer Aminogruppe am C-Atom Nr. 2. Wegen der näheren Verwandtschaft von Hypoxanthin zu Adenin erscheint somit eine leichtere Umwandelbarkeit in dieses möglich, außerdem muß aber auch in geringem Maße eine Umwandlung von Hypoxanthin in Guanin stattfinden.

Wie bereits oben erwähnt wurde, wird durch $\mathrm{Zu}$ satz von nicht markiertem Adenin zum Nährmedium die Aktivität des isolierten Guanins nicht verkleinert, woraus wir schlossen, daß eine Umwandlung von Adenin in Guanin nicht stattfindet. Durch Verwendung von Adenin ${ }^{-14} \mathrm{C}$ konnten wir diesen Beweis auf direktem Wege führen. Aus Tab. 2 geht hervor, daß in diesem Fall die Aktivität des eingebauten Guanins Null ist, während die des isolierten Adenins noch $46 \%$ der des eingesetzten Adenins beträgt. Dieses Adenin stammt somit aus dem zugesetzten markier-

8 M. F lavin u. S. Graff, J. biol. Chemistry 191. 55 [1951]; 192, 485 [1951]. ten Adenin und aus zugesetztem und im Bakterium synthetisiertem Guanin.

Um weiteren Einblick in die Umwandlung von Guanin in Adenin zu erhalten, haben wir auch Versuche mit Guanin- $\left(2-{ }^{14} \mathrm{C}\right)$ begonnen, über die später berichtet werden soll. Ferner ist an die Verwendung von Xanthin- $\left({ }^{14} \mathrm{C}\right)$ und Hypoxanthin- $\left({ }^{14} \mathrm{C}\right)$ gedacht.

Ganz ähnliche Ergebnisse, wie wir sie bei L. leichmannii 313 gefunden haben, sind bei Tetrahymena geleii $\mathrm{H}$ erhalten worden ${ }^{8}$. Dieses Protozoon benötigt ebenfalls Guanin als einziges Purin und Uracil als einziges Pyrimidin. Es findet eine Umwandlung von Guanin in Adenin statt, dagegen wird Adenin nicht in Guanin übergeführt. Bei Lactobacillus casei hingegen ist die gegenseitige Umwandlung von Guanin und Adenin möglich ${ }^{9}$.

9 M. E. B a lis, G. B. B rown, G. B. Elion, H. G. Hitchings u. H. Van der Werff, J. biol. Chemistry 188, 217 [1951].

\title{
Geiger-Müller'sche Miniatur-Zählrohre
}

\author{
Von W. Dreblow und W. Stremme \\ Aus dem Max-Planck-Institut für Biophysik, Frankfurt a. M. (Dir.: Prof. Dr. B. R a je w s k y) \\ (Z. Naturforschg. 7 b, 161-163 [1952]; eingegangen am 31. Dezember 1951) \\ Konstruktion, technische Daten, Lebensdauer und Lagerfähigkeit von Geiger-Müllerschen \\ Miniatur-Zählrohren, die sich zur Einführung in Körperhöhlen eignen, werden beschrieben.
}

$\mathrm{M}$ it der zunehmenclen Anwendung von Radioisotopen in der Medizin für diagnostische und therapeutische Zwecke werden die Forderungen nach einem GeigerMüllerschen Zählrohr spürbar, dessen Dimensionen so bemessen sein müssen, daß ein Einführen in Körperhöhlen möglich ist.

An dieser Stelle soll über Zählrohre berichtet werden, welche diese Forderung erfüllen und deren technische Daten ein einwandfreies Arbeiten immer dort gestatten, wo Geiger-Müllersche Zählrohre mit kleinem empfindlichen Volumen Verwendung finden.

Es ist bekannt, daß, je geringer der Kathodendurchmesser und je kürzer die empfindliche Länge des Zähldrahtes wird, sich die Eigenschaften eines Zählrohres verschlechtern. Der Geiger-Bereich und damit das Plateau werden kürzer, die Steigung nimmt zu und die Anzahl der spontanen Entladungen wird häufiger. Die Lebensdauer nimmt in ganz erheblichem Maße ab, ebenso die Lagerfähigkeit.

Es wurde eine Anzahl von Zählrohren hergestellt, die in diesen Beziehungen bedeutend verbesserte Eigenschaften aufweisen (Tab. 1).

Die Kathode besteht bei all diesen Zählrohren aus gezogenem Aluminiumrohr mit einer Wandstärke von $0,15 \mathrm{~mm}$, der Zähldraht aus technisch reinem Platin mit einem Durchmesser von 0,1 mm. Der Abschluß geschieht einerseits durch eine genau passende Glasperle, in welcher der Zähldraht eingeschmolzen ist. Auf der anderen Seite durch eine sich verjüngende Glaskapillare. Ein besonderes Verfahren gestattet ein Abschmelzen an dieser Stelle nach vorangehendem Evakuieren und Füllen, wobei der Zähldraht exakt zentriert werden kann und gespannt bleibt. Dies ist insofern von Bedeutung, als daß gerade bei Geiger-Müllerschen Zählrohren solch geringer

\begin{tabular}{|c|c|c|c|c|c|c|}
\hline & 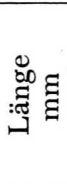 & 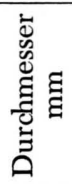 & 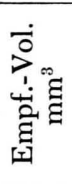 & 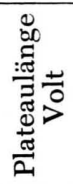 & 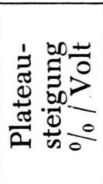 & 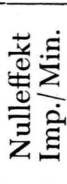 \\
\hline I & 30 & 5 & 490 & 150 & 0,35 & 3 \\
\hline II & 20 & 5 & 294 & 140 & 0,25 & 2 \\
\hline III & 30 & 4 & 314 & 150 & 0,3 & 3 \\
\hline IV & 20 & 3 & 120 & 110 & 0,3 & 1 \\
\hline
\end{tabular}

Tab. 1. Daten verschiedener Zählrohre (vgl. die Kennlinien, Abb. 3). 